\title{
Stationary Zonal Flows during the Formation of the Edge Transport Barrier in the JET Tokamak
}

\author{
J. C. Hillesheim, ${ }^{1, \dagger}$ E. Delabie, ${ }^{2}$ H. Meyer, ${ }^{1}$ C. F. Maggi, ${ }^{1}$ L. Meneses, ${ }^{3}$ E. Poli, ${ }^{4}$ and JET Contributors ${ }^{*}$ \\ EUROfusion Consortium, JET, Culham Science Centre, Abingdon, Oxon OX14 3DB, United Kingdom \\ ${ }^{1}$ CCFE, Culham Science Centre, Abingdon, Oxon OX14 3DB, United Kingdom \\ ${ }^{2}$ Oak Ridge National Laboratory, Oak Ridge, Tennessee, USA \\ ${ }^{3}$ Instituto de Plasmas e Fusão Nuclear, Instituto Superior Técnico, Universidade de Lisboa, Lisboa, Portugal \\ ${ }^{4}$ Max-Planck-institut fur Plasmaphysik, Garching, Germany \\ (Received 21 October 2015; published 10 February 2016)
}

\begin{abstract}
High spatial resolution Doppler backscattering measurements in JET have enabled new insights into the development of the edge $E_{r}$. We observe fine-scale spatial structures in the edge $E_{r}$ well with a wave number $k_{r} \rho_{i} \approx 0.4-0.8$, consistent with stationary zonal flows, the characteristics of which vary with density. The zonal flow amplitude and wavelength both decrease with local collisionality, such that the zonal flow $E \times B$ shear increases. Above the minimum of the $L-H$ transition power threshold dependence on density, the zonal flows are present during $L$ mode and disappear following the $H$-mode transition, while below the minimum they are reduced below measurable amplitude during $L$ mode, before the $L-H$ transition.
\end{abstract}

DOI: 10.1103/PhysRevLett.116.065002

Introduction.-The transition from low confinement ( $L$ mode) to high confinement ( $H$ mode) in tokamaks occurs due to the formation of a transport barrier near the plasma boundary - the pedestal - where the pressure gradient becomes large. This improves the global energy confinement time by about a factor of 2 , which is essential for achieving high fusion gain in future devices like ITER, the international experiment currently under construction that aims to demonstrate net production of fusion energy. Many models for the $L-H$ transition have been put forward (for reviews see Refs. [1-3]; for more recent work see Refs. [4-9]), but a validated theory has not been identified. The $L-H$ transition is usually characterized by a threshold in input heating power, $P_{L H}$. An enhanced understanding of the physics underlying the transition would improve predictions for $P_{L H}$ and possibly open routes to purposefully lower its value.

$H$-mode conditions were discovered in ASDEX more than 30 years ago [10,11]. It was quickly identified that the development of large shear in the radial electric field plays an important role in the $L-H$ transition [12-15] and that the transition is concurrent with a large drop in the amplitude of long wavelength density fluctuations [16-18]. There has been significant interest in recent years on the role of oscillatory zonal flows (toroidally and poloidally

Published by the American Physical Society under the terms of the Creative Commons Attribution 3.0 License. Further distribution of this work must maintain attribution to the author(s) and the published article's title, journal citation, and DOI. symmetric potential structures, $n=0, m=0$, with finite radial wave numbers) in $L-H$ transition dynamics [19-23] in the form of the geodesic acoustic mode (GAM) and low frequency "limit cycle oscillations" (LCOs). It has also been reported that in some cases the turbulence drive through the measured Reynolds stress is too small to account for the amplitude of LCOs [24].

We report high spatial resolution measurements of the radial electric field, $E_{r}$, with Doppler backscattering (DBS) in JET, which reveals fine-scale spatial structure in $E_{r}$ that can be stationary for $100 \mathrm{~s}$ of $\mathrm{ms}$. This stationarity is consistent with the predicted long time behavior for zonal flows (ZFs) in tokamaks $[25,26]$, while until now only low-but finite-frequency ZFs have been reported in experiments [19-23,27-30]. This implies a qualitative difference in the interaction between turbulence and the ZFs, since in addition to representing an energy sink, stationary ZFs can more effectively regulate turbulence through $E \times B$ shearing. See Ref. [31] for a review of ZF physics in plasmas.

These stationary zonal flows in JET are only observed, so far, in the $E_{r}$ well and before the $L-H$ transition. ZFs have been predicted to be weak or absent in the pedestal region $[32,33]$. It has been well established that there is a nonmonotonic dependence of $P_{L H}$ on density [34-39], which has also been found in JET with the ITER-like $\mathrm{W} / \mathrm{Be}$ wall [40]. It has been hypothesized that this is related to a decoupling of the ion and electron heat fluxes due to a requirement on only the ion heat flux for the transition [41]; the empirical prediction for the density minimum in Ref. [41] agrees reasonably with JET data in some divertor configurations [42]. We report that ZFs are present until the 
$L-H$ transition in the high density branch, after which they are below measurable amplitudes. In the low density branch, the ZFs reduce below measurable amplitude long before the $L-H$ transition. The wavelength of the ZFs scales inversely with density. Zonal flows are predicted to have finite radial wave numbers of order $k_{r, \mathrm{ZF}} \rho_{i} \sim 0.1$ (where $\rho_{i}$ is the ion gyroradius), but little attention has been given to dependencies of $k_{r, \mathrm{ZF}}$.

The experiment.-Measurements were obtained during experiments in JET studying dependencies of $P_{L H}$. The experiment was performed in a neutral beam injection (NBI) heated plasma with toroidal field $B_{\phi}=3 \mathrm{~T}$ and plasma current $I_{p}=2.5 \mathrm{MA}$, with $q_{95} \approx 3.4$. In one divertor configuration using a vertically up-shifted plasma the alignment of a microwave diagnostic system [43] designed for normal-incidence correlation reflectometry measurements changed sufficiently that DBS measurements were obtained instead. This shape has a $P_{L H}$ value that is about a factor of 2 higher than other configurations in JET [42]. The NBI power was slowly ramped up to about 10 MW over 7 seconds to identify $P_{L H}$. The line-averaged density was varied shot to shot from $\left\langle n_{e}\right\rangle=1.6 \times 10^{19} \mathrm{~m}^{3}$ to $3.1 \times 10^{19} \mathrm{~m}^{3}$. In JET the ion and electron temperatures are equal within uncertainties even in the low density branch of the transition $[44,45]$. There was a mode at about $10 \mathrm{kHz}$ identified as the GAM [46], but it was only present during the Ohmic phase and was not of measurable amplitude during the NBI-heated $L$-mode or $H$-mode time periods. Directly after the $L-H$ transition, the plasma enters an edge localized mode (ELM)-free $H$ mode with an $m=1, n=0$ magnetic oscillation, with frequency 1-2 kHz, at the top of the pedestal that has been named " $M$ mode" [47]. In this Letter we focus on the the mean electric field and its structure.

The DBS technique [48] yields measurements of the propagation velocity of turbulent structures, $v_{\text {turb }}$, and density fluctuation levels. The TORBEAM beam tracing code [49] is used with the reconstructed magnetic equilibrium from EFIT and density profiles from a profile reflectometer [50] (averaged over the period of time the $E_{r}$ profile is obtained) as inputs to determine the local scattering position and wave number. Since $v_{\text {turb }}=$ $v_{E \times B}+v_{\mathrm{ph}}$, if the phase velocity of the turbulence $v_{\mathrm{ph}}$ is small, then the measured velocity is dominated by the $v_{E \times B}$ drift and $E_{r}$ can be inferred. One would expect the linear phase velocity to be on the order of the diamagnetic velocity, but could be in either the ion or electron direction depending on the nature of the turbulence. The DBS measurements are obtained at low wave number, $k_{\perp} \approx 2.5-3.0 \mathrm{~cm}^{-1}$. The comparison below was performed to cross-check measurement fidelity and assess the magnitude of $v_{\text {ph }}$.

Figure 1 compares $E_{r}$ measured with DBS and with a charge exchange recombination spectroscopy (CXRS) diagnostic measuring carbon impurities, for a time window

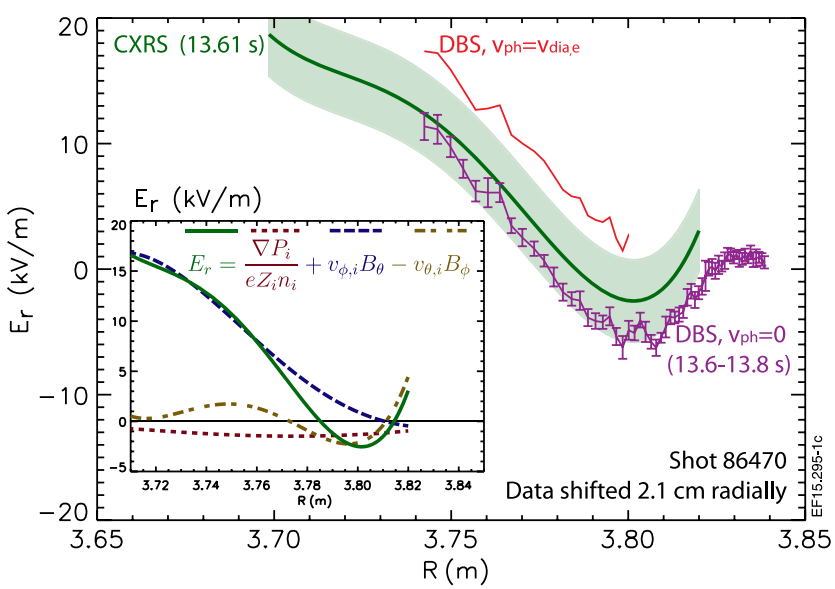

FIG. 1. Comparison of $E_{r}$ measured with CXRS (uncertainties indicated by shaded area) and DBS in $L$ mode. DBS data shown both assuming $v_{\mathrm{ph}}=0$ and $v_{\mathrm{ph}}=v_{\mathrm{dia}, e}$. DBS data are shifted radially by $2.1 \mathrm{~cm}$. Shown in the inset panel are the the polynomial fits for each of the CXRS $E_{r}$ terms and their sum.

where CXRS had relatively low uncertainties. The DBS data for a major radius $R<3.75 \mathrm{~m}$ are affected by aliasing, since the diagnostic was not originally intended for DBS measurements and was digitized at $2 \mathrm{MHz}$; aliased data are omitted. For $R>3.75 \mathrm{~m}$, we find good agreement for the profile shape after a radial shift has been applied. The radial shift is partially explained by a $\sim 10 \mathrm{~cm}$ offset in the vertical position of the measurements, with the remainder likely due to $\sim 1 \mathrm{~cm}$ uncertainty in the location of the separatrix in the reconstruction. The DBS measurements here were acquired with a single frequency source, which was adjusted by $200 \mathrm{MHz}$ every $2.5 \mathrm{~ms}$ with a switching time of $\sim 60 \mu \mathrm{s}$, to acquire a profile over 150 or $200 \mathrm{~ms}$. The spectra for the CXRS data were averaged over $20 \mathrm{~ms}$, corresponding to a time near the start of the DBS sweep. The small radial spacing between the DBS measurements enables structures smaller than the radial resolution of a single CXRS channel $(\sim 2-4 \mathrm{~cm})$ to be resolved. The inset in Fig. 1 shows the polynomial fits for the individual components from CXRS and their sum. The pressure term is small due to measuring an impurity. The toroidal flow dominates force balance in the core, and also contributes significantly in the $E_{r}$ well region, which has been previously observed in JET [40]. The poloidal velocity would be expected to be neoclassical, following the main ion pressure gradient [51]. Profiles assuming either $v_{\mathrm{ph}}=0$ or $v_{\mathrm{ph}}=v_{\mathrm{dia}, e}$ are plotted, where $v_{\mathrm{dia}, e}=\mathbf{B} \times \nabla P_{e} /\left(e B n_{e}\right)$ is the electron diamagnetic velocity determined from profile reflectometry and electron cyclotron emission; optically thin data omitted. Comparing DBS and CXRS, the data are consistent with $0 \lesssim v_{\mathrm{ph}} \lesssim v_{\mathrm{dia}, e}$ with best agreement in the well region for $v_{\mathrm{ph}} \approx v_{\mathrm{dia}, e} / 2$; lacking a specific prediction for the value of $v_{\mathrm{ph}}$, we take it to be zero in later plots. Later comparisons also imply a finite $v_{\mathrm{ph}}$ in some cases, of similar magnitude to the CXRS uncertainty. 


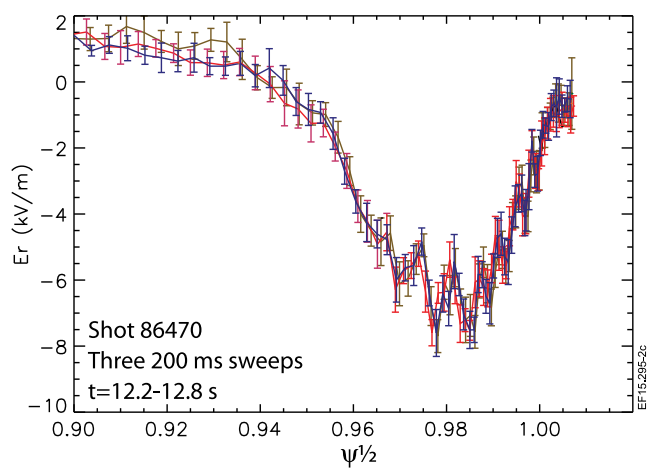

FIG. 2. $E_{r}$ profile measured with three consecutive $200 \mathrm{~ms}$ sweeps during a steady-state Ohmic time period.

If the kinetic profiles vary smoothly then the fine scale structure in $v_{\text {turb }}$ can be attributed to $v_{E \times B}$; however, structure in $v_{\mathrm{ph}}$ cannot be assessed directly from the DBS vs CXRS comparison and there could be structure in the temperature profiles below the measurement resolution. Even if there is structure in $v_{\mathrm{ph}}$, it could still be related to the temperature profile structure predicted to accompany ZFs in some regimes [33,52].

Fine-scale spatial structure in $E_{r}$. -Figure 2 shows three consecutive radial profiles of $E_{r}$ inferred from DBS during a steady-state Ohmic time period. There is fine-scale structure in the profile, with static radial oscillations that persist during the $600 \mathrm{~ms}$ window. The measurements are highly reproducible and the spatial structures are larger than the error bars (standard deviation within each $2.5 \mathrm{~ms}$ step). These stationary structures are largest at the bottom of the $E_{r}$ well, which corresponds to a large pressure gradient. The fine-scale structure varies with plasma conditions at fixed safety factor profile, shown in Fig. 3, so it cannot be attributed to magnetic islands that would be

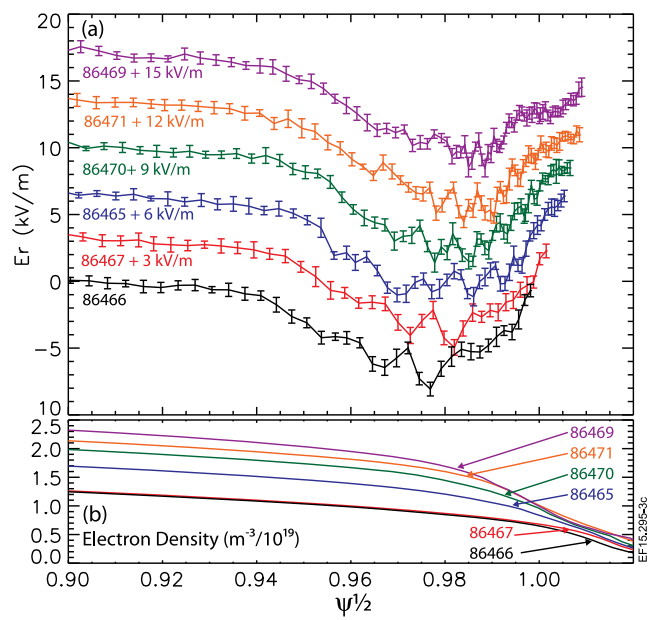

FIG. 3. (a) Ohmic $E_{r}$ profiles for six shots at different densities, $t=12.4-12.6 \mathrm{~s}$. For clarity, for each density increment the $E_{r}$ profile is offset by an additional $3 \mathrm{kV} / \mathrm{m}$ (annotated). (b) Averaged $n_{e}$ profile $12.4-12.6 \mathrm{~s}$, from a profile reflectometer. related to rational surfaces. Although with measurements at only one toroidal location we cannot confirm symmetry properties directly, since the structures are static in both space and time at radii with finite rotation, $n=0$ structure is strongly implied. With alternative interpretations contradicted and with measurable expectations for ZFs satisfied, we identify the fine-scale structure in $E_{r}$ as zonal flows. In some shots small differences in the locations of peaks and troughs are observed, but this could be due to small changes in kinetics profiles or equilibrium, and we have no evidence for radial propagation.

The ZFs show variation with density, which is seen most clearly during the Ohmic phase. Figure 3 shows the $E_{r}$ profile and averaged density during a $200 \mathrm{~ms}$ steady-state Ohmic time window. As the density rises, the wavelength of the ZFs decreases and their region of existence moves outward. The width of the $E_{r}$ well also decreases with density and the core $E_{r}$ monotonically increases from about $0 \mathrm{kV} / \mathrm{m}$ for the lowest density to about $2.5 \mathrm{kV} / \mathrm{m}$ for the highest, at $\sqrt{\psi} \approx 0.90$, where $\psi$ is the normalized poloidal flux.

Parametric scaling of zonal flows.-With the present limited data we cannot conclusively identify parametric scalings; however, we can compare to expectations. The amplitude, $V_{\mathrm{ZF}}$, and radial wavelength, $\lambda_{\mathrm{ZF}}$, of the ZFs are directly determined from the bottom of the $E_{r}$ well in Fig. 3, and plotted as a function of the local collisionality, $\nu_{*}=q R \nu_{i i} /\left(v_{t h, i} e^{3 / 2}\right)$, in Fig. 4, where $V_{\mathrm{ZF}}$ is half the peak-to-peak amplitude, $\nu_{i i}$ is the ion collision rate, and $\epsilon=r / R$ is the local inverse aspect ratio ( $r$ is the local minor radius). There is also a monotonic increase of $\lambda_{\mathrm{ZF}}$ with $\rho_{i}$, but $\rho_{i}$ only changes by about $10 \%$, and $k_{r, \mathrm{ZF}} \rho_{s}$ spans $0.35-0.85$, so the ZF wavelength is not simply changing to keep $k_{r, \mathrm{ZF}} \rho_{i}$ constant. Figure 4 shows the scaling with collisionality, as ion collisions are expected to damp zonal flows. There is little trend for $V_{\mathrm{ZF}}$, while there is a clear decrease of $\lambda_{\mathrm{ZF}}$ with $\nu_{*}$; however, $\nu_{*}$ values cross from the banana to plateau regime, which could change the collisional regime for ZF damping [31]. The reduction of $\lambda_{\mathrm{ZF}}$ is larger than the changes to $V_{\mathrm{ZF}}$, such that the zonal flow

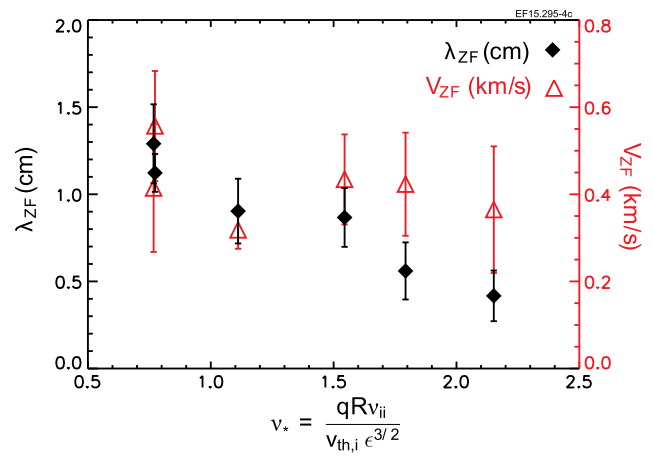

FIG. 4. Local scaling of the zonal flow radial wavelength and amplitude with collisionality. 
shear, $\sim V_{\mathrm{ZF}} / \lambda_{\mathrm{ZF}}$, increases with collisionality. This implies that arguments based only on collisional damping of ZFs, while ignoring $k_{r, \mathrm{ZF}}$, may be misleading.

Changes across the L-H transition.-Figure 5 shows the changes to $E_{r}$ and density fluctuation before and after the $L-H$ transition, identified by changes to $D_{\alpha}$ emission and the $T_{i}$ profile, at several densities. The lowest density is below the minimum in the density dependence of $P_{L H}$, while the other two are above. Since fast dynamics are only captured for a single point, the profile during which the transition occurs is omitted; a period of unsustained transitions in 86467 is also omitted. For several hundred milliseconds before and after the $L-H$ transition, the $E_{r}$ profile at the edge is insensitive to the slow NBI power ramp. At the lowest density, the amplitude of the ZFs is already reduced to below measurable levels during $L$ mode, well before the $L-H$ transition, while there is a reduction in the $\mathrm{ZF}$ amplitude across the transition at high densities, observed most clearly in Fig. 5(b). Shown in the insets in Figs. 5(a)-5(c) are the $E_{r}$ profiles from CXRS, with polynomial fits averaged over the DBS sweep before and after the transition; different abscissa units are used due to the unknown radial offset, discussed above. At high densities we observe a clear increase in the minimum of the $E_{r}$ well inferred from DBS when assuming $v_{\mathrm{ph}}=0$ (i.e., the well becomes shallower), which is not observed at low density. Although the changes are of similar magnitude to CXRS uncertainties, the CXRS $E_{r}$ in both high density cases changes in the opposite direction to that observed with DBS, suggesting the change to the DBS profile is due to $v_{\mathrm{ph}}$.

The density fluctuation levels $\delta n / n$ at $k_{\perp} \rho_{i} \approx 0.2$ measured with DBS are shown in Figs. 5(d)-5(f), normalized to a time window during the steady-state Ohmic period. For $\sqrt{\psi}<0.95, \delta n / n$ falls during $L$ mode, as a large $E \times B$ shear is driven by the NBI. At high densities, there is a clear drop in $\delta n / n$ by $20 \%-30 \%$ after the transition in the well region, $0.95 \lesssim \sqrt{\psi} \lesssim 0.99$. At low density, a more limited drop is observed $0.97 \lesssim \sqrt{\psi} \lesssim 0.99$, and a slight increase is observed from Ohmic to $L$ mode at $\sqrt{\psi} \approx 0.97$. It is notable that the drop in $\delta n / n$ across the $L-H$ transition does not appear to be related to an increase in $E \times B$ shear comparable to the change from Ohmic to $L$ mode. Since DBS measurements can be affected by nonlinear saturation [53,54], observations are a lower bound on changes to $\delta n / n$ and lack of observed change at $\sqrt{\psi} \gtrsim 0.99$ could be due to saturation. These results imply that a collapse of the ZF amplitude and turbulence phase velocity, along with the fluctuation amplitude, is important for the turbulence regime in the high density branch of the $L-H$ transition, but not in the low density branch. This is consistent with a fundamental difference in the turbulence regime in the two branches.

Conclusions.-High spatial resolution DBS measurements have revealed novel insights into the development of the edge transport barrier in JET. For the first time, finescale structures in the $E_{r}$ profile consistent with static zonal flows have been observed in a tokamak. These zonal flows are analogous to the banded structures and jet streams observed in planetary atmospheres [55]. In JET, they appear at the bottom of the edge $E_{r}$ well. This is a significant observation, implying that stationary ZFs are what is important for development of the pedestal in JET, rather than the GAMs and LCOs observed in other experiments. The ZFs are reduced below measurable amplitude in $H$ mode. The different observations at high and low density also suggest a possible relation to the nonmonotonic behavior of $P_{L H}$.
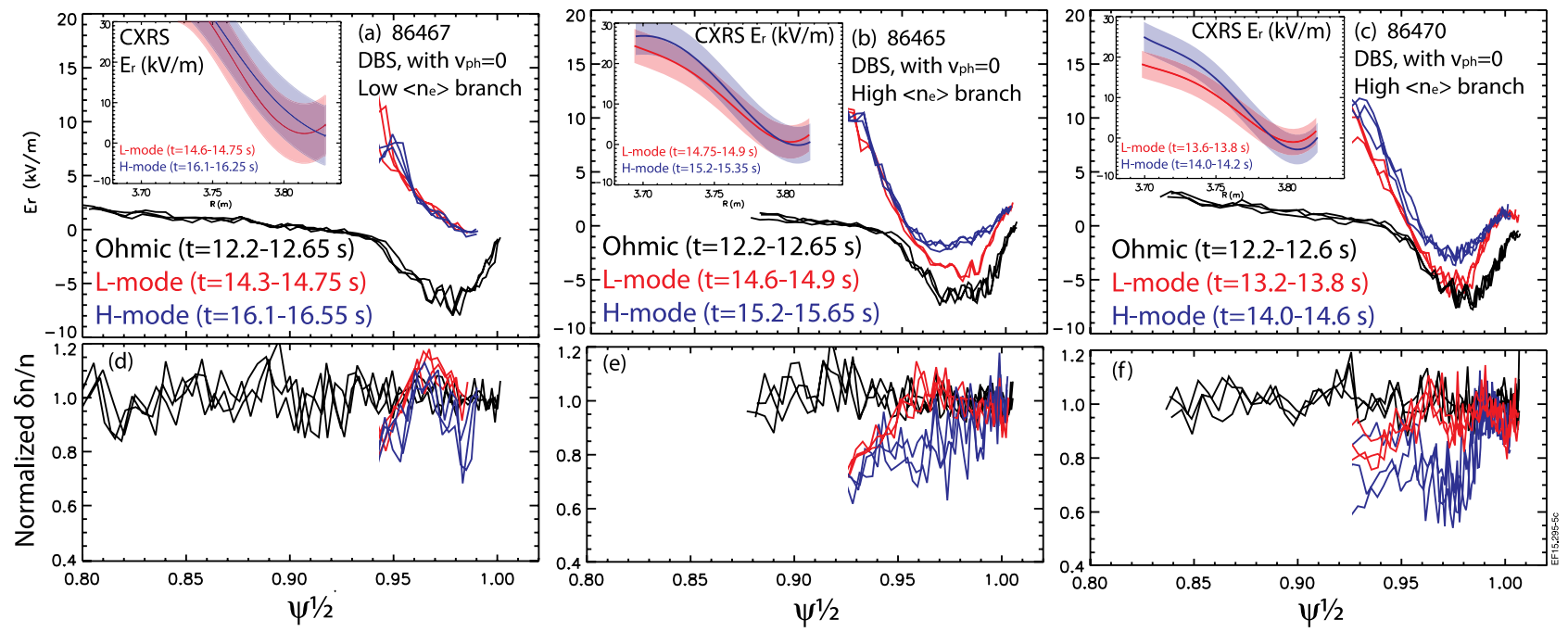

FIG. 5. (a)-(c) $E_{r}$ and density fluctuation (d)-(f) profiles for Ohmic conditions and several hundred milliseconds before and after the $L-H$ transition at three densities: (a),(d) $\left\langle n_{e}\right\rangle=1.6 \times 10^{19} \mathrm{~m}^{-3}$, (b), (e) $\left\langle n_{e}\right\rangle=2.0 \times 10^{19} \mathrm{~m}^{-3}$, and (c), (f) $\left\langle n_{e}\right\rangle=2.6 \times 10^{19} \mathrm{~m}^{-3}$. Density fluctuations measured at $k_{\perp} \approx 3 \mathrm{~cm}^{-1}\left(k_{\perp} \rho_{i} \approx 0.2\right)$ and normalized to Ohmic values. 
In JET there can be a well-defined $E_{r}$ well even in Ohmic plasmas, instead of the well only forming after the $L-H$ transition. For the configuration studied here, with a high $P_{L H}$, the NBI power required to reach the transition already results in large $E \times B$ shear and initial reduction in fluctuation amplitudes near the edge during $L$ mode, rather than only after the transition. These observations separate necessary conditions for sustaining the $H$-mode pedestal from the causes of the $L-H$ transition and its effects, and aid in discriminating between models for the transition. For projection to larger devices like ITER, it is important to understand whether these observations are unique to the $\mathrm{W} / \mathrm{Be}$ wall in JET, to the divertor configuration, to high $P_{L H}$ with NBI heating, or whether they are universal in character, motivating further experimental and theoretical work.

This work has been carried out within the framework of the EUROfusion Consortium and has received funding from the Euratom research and training programme 2014-2018 under Grant agreement No. 633053. The views and opinions expressed herein do not necessarily reflect those of the European Commission.

*See the Appendix of F. Rommanelli et al., Proceedings of the 25th IAEA Fusion Energy Conference 2014, Saint Petersburg, Russia. †jon.hillesheim@ccfe.ac.uk

[1] K. H. Burrell et al., Plasma Phys. Controlled Fusion 34, 1859 (1992).

[2] J. W. Connor and H. R. Wilson, Plasma Phys. Controlled Fusion 42, R1 (2000).

[3] E. J. Doyle et al., Nucl. Fusion 47, S18 (2007).

[4] W. Fundamenski, F. Militello, D. Moulton, and D.C. McDonald, Nucl. Fusion 52, 062003 (2012).

[5] E. R. Solano and R. D. Hazeltine, Nucl. Fusion 52, 114017 (2012).

[6] K. Miki, P. H. Diamond, S.-H. Hahn, W. W. Xiao, Ö. D. Gürcan, and G. R. Tynan, Phys. Rev. Lett. 110, 195002 (2013).

[7] C. Bourdelle et al., Nucl. Fusion 54, 022001 (2014).

[8] G. M. Staebler and R. J. Groebner, Plasma Phys. Controlled Fusion 57, 014025 (2015).

[9] X. Wu, G. Xu, B. Wan, J. J. Rasmussen, V. Naulin, and A. H. Nielsen, Nucl. Fusion 55, 053029 (2015).

[10] F. Wagner et al., Phys. Rev. Lett. 49, 1408 (1982).

[11] F. Wagner et al., Phys. Rev. Lett. 53, 1453 (1984).

[12] R. J. Taylor, M. Brown, B. Fried, H. Grote, J. Liberati, G. Morales, P. Pribyl, D. Darrow, and M. Ono, Phys. Rev. Lett. 63, 2365 (1989).

[13] H. Biglari, P. H. Diamond, and P. W. Terry, Phys. Fluids B 2, 1 (1990).

[14] R. J. Groebner, K. H. Burrell, and R. P. Seraydarian, Phys. Rev. Lett. 64, 3015 (1990).

[15] K. H. Burrell, Phys. Plasmas 6, 4418 (1999).

[16] E. J. Doyle, R. J. Groebner, K. H. Burrell, P. Gohil, T. Lehecka, N. C. Luhmann, H. Matsumoto, T. H. Osborne,
W. A. Peebles, and R. Philipona, Phys. Fluids B 3, 2300 (1991).

[17] H. Matsumoto et al., Plasma Phys. Controlled Fusion 34, 615 (1992).

[18] G. R. Tynan, L. Schmitz, R. W. Conn, R. Doerner, and R. Lehmer, Phys. Rev. Lett. 68, 3032 (1992).

[19] G. D. Conway, C. Angioni, F. Ryter, P. Sauter, and J. Vicente, Phys. Rev. Lett. 106, 065001 (2011).

[20] T. Estrada, C. Hidalgo, T. Happel, and P. H. Diamond, Phys. Rev. Lett. 107, 245004 (2011).

[21] G. S. Xu et al., Phys. Rev. Lett. 107, 125001 (2011).

[22] L. Schmitz, L. Zeng, T. L. Rhodes, J. C. Hillesheim, E. J. Doyle, R. J. Groebner, W. A. Peebles, K. H. Burrell, and G. Wang, Phys. Rev. Lett. 108, 155002 (2012).

[23] G. R. Tynan et al., Nucl. Fusion 53, 073053 (2013).

[24] T. Kobayashi et al., Phys. Rev. Lett. 111, 035002 (2013).

[25] M. N. Rosenbluth and F. L. Hinton, Phys. Rev. Lett. 80, 724 (1998).

[26] F. L. Hinton and M. N. Rosenbluth, Plasma Phys. Controlled Fusion 41, A653 (1999).

[27] A. Fujisawa et al., Phys. Rev. Lett. 93, 165002 (2004).

[28] D. K. Gupta, R. J. Fonck, G. R. McKee, D. J. Schlossberg, and M. W. Shafer, Phys. Rev. Lett. 97, 125002 (2006).

[29] G. S. Xu et al., Phys. Plasmas 19, 122502 (2012).

[30] P. Manz et al., Phys. Plasmas 19, 072311 (2012).

[31] P. H. Diamond, S.-I. Itoh, K. Itoh, and T. S. Hahm, Plasma Phys. Controlled Fusion 47, R35 (2005).

[32] B. Scott, Phys. Lett. A 320, 53 (2003).

[33] G. Dif-Pradalier et al., Phys. Rev. Lett. 114, 085004 (2015).

[34] S. J. Fielding, J. D. Ashall, P. G. Carolan, A. Colton, D. Gates, J. Hugill, A. W. Morris, M. Valovic, the COMPASS-D, and ECRH teams, Plasma Phys. Controlled Fusion 38, 1091 (1996).

[35] T. N. Carlstrom and R. J. Groebner, Phys. Plasmas 3, 1867 (1996).

[36] L. D. Horton et al., Proc. 26th EPS Conf. on Conrolled Fusion and Plasma Physics, Maastricht, The Netherlands (1999).

[37] Y. Andrew et al., Plasma Phys. Controlled Fusion 48, 479 (2006).

[38] F. Ryter et al., Nucl. Fusion 49, 062003 (2009).

[39] Y. Ma, J. W. Hughes, A. E. Hubbard, B. LaBombard, R. M. Churchill, T. Golfinopolous, N. Tsujii, and E. S. Marmar, Nucl. Fusion 52, 023010 (2012).

[40] C. F. Maggi et al., Nucl. Fusion 54, 023007 (2014).

[41] F. Ryter, L. Barrera Orte, B. Kurzan, R. M. McDermott, G. Tardini, E. Viezzer, M. Bernert, and R. Fischer, Nucl. Fusion 54, 083003 (2014).

[42] H. Meyer et al., in Proceeding of the 41st European Physics Society Conference on Plasma Physics, Berlin, P1.013 (2014).

[43] A. Sirinelli et al., in Proceeding of the 11th Intl. Reflectometry Workshop-IRW11, Palaiseau, France (2013).

[44] E. Delabie et al., in Proceeding of the 25th IAEA Fusion Energy Conference, Saint Petersburg, Russia, EX/P5 (2014).

[45] E. Delabie et al., in Proceeding of the 42nd European Physics Society Conference on Plasma Physics, Lisbon, O3.113 (2015). 
[46] C. Silva et al., in Proceeding of the 42nd European Physics Society Conference on Plasma Physics, Lisbon, O3.114 (2015).

[47] E. R. Solano et al., in Proceeding of the 40th European Physics Society Conference on Plasma Physics, Espoo, P4.111 (2013).

[48] M. Hirsch and E. Holzhauer, Plasma Phys. Controlled Fusion 46, 593 (2004).

[49] E. Poli, A. G. Peeters, and G. V. Pereverzev, Comput. Phys. Commun. 136, 90 (2001).

[50] A. Sirinelli et al., Rev. Sci. Instrum. 81, 10D939 (2010).
[51] E. Viezzer, T. Pütterich, C. Angioni, A. Bergmann, R. Dux, E. Fable, R. M. McDermott, U. Stroth, and E. Wolfrum, Nucl. Fusion 54, 012003 (2014).

[52] G. Dif-Pradalier, P. H. Diamond, V. Grandgirard, Y. Sarazin, J. Abiteboul, X. Garbet, Ph. Ghendrih, A. Strugarek, S. Ku, and C. S. Chang, Phys. Rev. E 82, 025401(R) (2010).

[53] E. Z. Gusakov, A. V. Surkov, and A. Y. Popov, Plasma Phys. Controlled Fusion 47, 959 (2005).

[54] U. Stroth et al., Nucl. Fusion 55, 083027 (2015).

[55] P. S. Marcus, T. Kundu, and C. Lee, Phys. Plasmas 7, 1630 (2000). 